\title{
A 3D Geometry-based Stochastic Model for 5G Massive MIMO Channels
}

\author{
(Invited Paper) \\ Yi Xie, Bo Li, Xiaoya Zuo, Mao Yang and Zhongjiang Yan \\ School of Electronics and Information, \\ Northwestern Polytechnical University \\ Email:xieyi@mail.nwpu.edu.cn \\ \{libo.npu, zuoxy, yangmao, zhjyan\}@nwpu.edu.cn
}

\begin{abstract}
Massive MIMO is one of the most promising technologies for the fifth generation (5G) mobile communication systems. In order to better assess the system performance, it is essential to build a corresponding channel model accurately. In this paper, a three-dimension (3D) two-cylinder regular-shaped geometry-based stochastic model (GBSM) for non-isotropic scattering massive MIMO channels is proposed. Based on geometric method, all the scatters are distributed on the surface of a cylinder as equivalent scatters. Non-stationary property is that one antenna has its own visible area of scatters by using a virtual sphere. The proposed channel model is evaluated by comparing with the 3GPP 3D channel model [1]. The statistical properties are investigated. Simulation results show that close agreements are achieved between the characteristics of the proposed channel model and those of the 3GPP channel model, which justify the correctness of the proposed model. The model has advantages such as good applicability.
\end{abstract}

Index Terms-5G, Massive MIMO, 3D GBSM, two-cylinder channel model, Non-stationary.

\section{INTRODUCTION}

With the proliferation of wireless services, $5 \mathrm{G}$ system puts higher requirements on its capacity and link rates. Multipleinput multiple-output (MIMO) [2] technologies significantly enhance the network throughput, which provides a technical guarantee for mobile communications [3], [4]. Recent studies show that massive MIMO system equipped with hundreds of antennas has significant advantages over the traditional MIMO technology [5], [6], and has become one of the most competitive technologies for $5 \mathrm{G}$.

A feasible $5 \mathrm{G}$ channel model should be established to practically evaluate the system performance. 5G massive MIMO channel model is different from the conventional channel model [7]. Firstly, the non-stationary property is found in the multi-antenna array [8], which means different antenna element can see different scatters, and the closer the two antennas sit, the more common scatters they share. Secondly, it's more practical to establish $5 \mathrm{G}$ channel models in a threedimension (3D) space [9]. Thirdly, spherical wave effect must be considered in massive MIMO array [5].

Most of the available studies model the MIMO channels in a two-dimensional space [10]-[12]. A two-dimensional model with single-ring or dual-ring is proposed in [11]. However it cannot accurately describe the real scene of the threedimensional space. The channel model WINNER adds the influence of elevation angle [12]. Moreover, The geometrybased stochastic models are widely used in mobile-to-mobile communication systems with equivalent scatters spreading on the surface. The GBSM is often applied in sphere models, but it is seldom used in infrastructure-based cellular networks. The indoor channel model in [13] describes a scene where the two spheres surrounding the antennas at the transmitter and receiver respectively are different in height. In [8], a theoretical non-stationary 3D twin-cluster channel model for massive MIMO communication systems is proposed. It uses a birthdeath process to model non-stationary properties of clusters, such as clusters appear and disappear on both the array and time axes. The channel model in [8] is valuable and effective. But many parameters in the model are difficult to match with values from actual measurements, and the geometric meanings of the model are not clear, which affects its applicabilities in 5G. Furthermore, because of the non-stationary property, the traditional MIMO channel model cannot be extended to massive MIMO, and a model which can be consistent with the general standards is needed. Based on these above considerations, a 3D GBSM method is adopted to model the massive MIMO channel in this paper, which is easy to implement.

In this paper, we propose a non-stationary $5 \mathrm{G}$ massive MIMO channel model based on GBSM. To the best of the authors' knowledge, this is the first work to use a two-cylinder GBSM model in a 3D space to characterize the non-stationary property in multi-antenna array. The contributions of this paper are summarized as follows:

- A two-cylinder GBSM method is established for 5G channel. In the proposed model,it is assumed that the azimuth angles cover 360 degrees in the horizontal direction when signal travels in space, while the elevation angles only cover a certain range in the vertical direction [14], which is more reasonable to use a two-cylinder model.

- The impacts of the non-stationary property are verified by using geometric method. The visible range of each antenna is denoted by a sphere, and equivalent scatters can be seen by a specific antenna element only if they are separated less than the radius of the sphere. Each antenna has its own sphere to establish its own set of scatters. 
- The approximate value of radius of the visible sphere is calculated. It describes the correlation between the channels of antenna elements. The correlation is determined by the number of the scatters they share. The concept of equivalent scatters is introduced at both the transmitter and the receiver. The antenna array is surrounded by a cylinder at each side. It's assumed that the local scatters in the vicinity are distributed on the column surface of the cylinder.

The rest of this paper is organized as follows. The properties of 5G massive MIMO channel and the geometric method to achieve non-stationary property are introduced in section II. In Section III, a 3D GBSM channel model for massive MIMO is proposed. Section IV studies the statistical properties of the proposed model. Simulation results and analysis are presented in Section $\mathrm{V}$, and the advantages of the proposed channel model are listed at last. Finally, conclusions are summarized in Section VI.

\section{NEW PROPERTIES IN MASSIVE MIMO CHANNEL MODELS}

It is very important and meaningful to choose an appropriate $5 \mathrm{G}$ channel model for the optimization and evaluation of the system performance. MIMO channel modeling methods are mainly based on their statistical properties. The observed fading phenomenons in MIMO channel are depicted by statistical averaging method. One of the typical methods is GBSM, which is widely used due to its convenience for theoretical analysis of channel statistics. In GBSMs, the propagation paths of the electromagnetic waves are modeled through randomly distributed local scatters rather than the specific channel environment. In consideration of the novelty and uniqueness of the 5G channel, we propose a 3D GBSM massive MIMO channel model, which includes three new properties: 3D, spherical wave effect and non-stationary property.

\section{A. 3D MIMO}

Most of the traditional MIMO channels are modeled in 2D, but massive MIMO arrays in $5 \mathrm{G}$ are going to be equipped with numerous antennas in more than one plane. There are different kinds of the antenna array configurations in 5G massive MIMO system, such as the rectangular, spherical and cylindrical antenna array deployments. Because of these MIMO antenna array deployments, signals travel in both azimuth and elevation angles, which makes the signal radiation more practical. So it is essential to model it in $3 \mathrm{D}$.

\section{B. Spherical Wave Effect}

When large antenna arrays are employed at massive MIMO system, plane wavefront assumptions in traditional channel models are inappropriate for the massive MIMO channel [5]. Spherical wave effect can accurately reflect the realistic channel properties and is more rational for massive MIMO channel modeling even with the higher computation complexity.

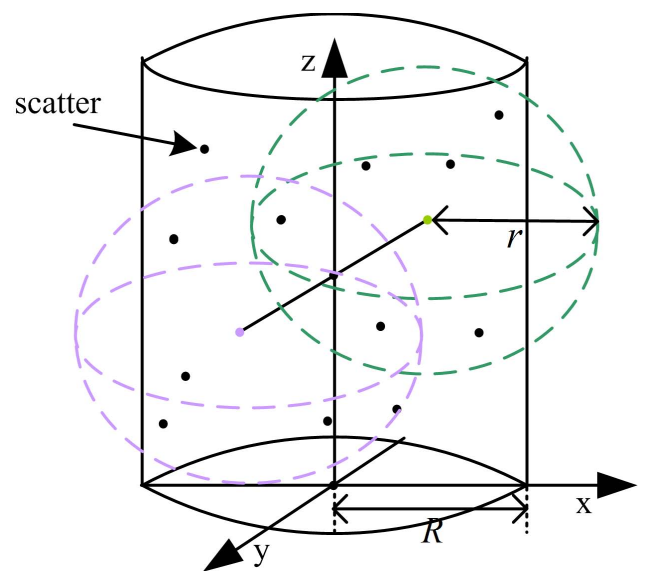

Fig. 1. Non-stationary property.

\section{Non-stationary Property}

Employing large antenna arrays, the non-stationary property have been observed through real world measurements [15], [16]. The non-stationary property refers to the fact that as an antenna array goes large, each antenna at different physical location has different perspective. GBSMs are often used to evaluate the performance of practical wireless communication systems in channel model standards, which accurately reflect the channel properties.

\section{1) Cylinder Model and Spherical Visible Area Model}

It's assumed that all the scatters existing in the vicinity can be replaced by the equivalent scatters on the column surface of a cylinder. For a large-scale antenna array in both the transmitter and the receiver, not all the scatters can be seen by each antenna element, and different antenna element can see different scatters. The closer the two antennas are, the more common scatters they share. In order to characterize the nonstationary property and determine the scatters which can be seen by a particular antenna, a geometric method is proposed based on a cylinder model and a spherical model, as it shows in Fig. 1.

In the proposed geometric model, the visible range of each antenna is denoted by a virtual sphere, where the radius is $r$ and the center is the antenna itself. In this model, scatters on the surface of the cylinder can be seen by an antenna only if the distance between them is less than $r$. Therefore, each antenna element has a different set of visible scatters. In this paper the sphere radii of the antennas in the transmitter and the receiver are denoted as $r_{t}$ and $r_{r}$, respectively.

\section{2) Radius of the Spherical Visible Area}

From the measurement results shown in [15], [16], we know that for a particular distance between two antenna elements, the correlation of their channel impulse responses can be seen as a constant value. The value reflects the grade of their sharing scatters. According to the distance and the correlation value, the radius of the sphere can be calculated.

It is assumed that there are two antenna elements, they are in the same horizontal level and symmetry with the central vertical axis of the cylinder, the distance between them is $\lambda / 2$ 


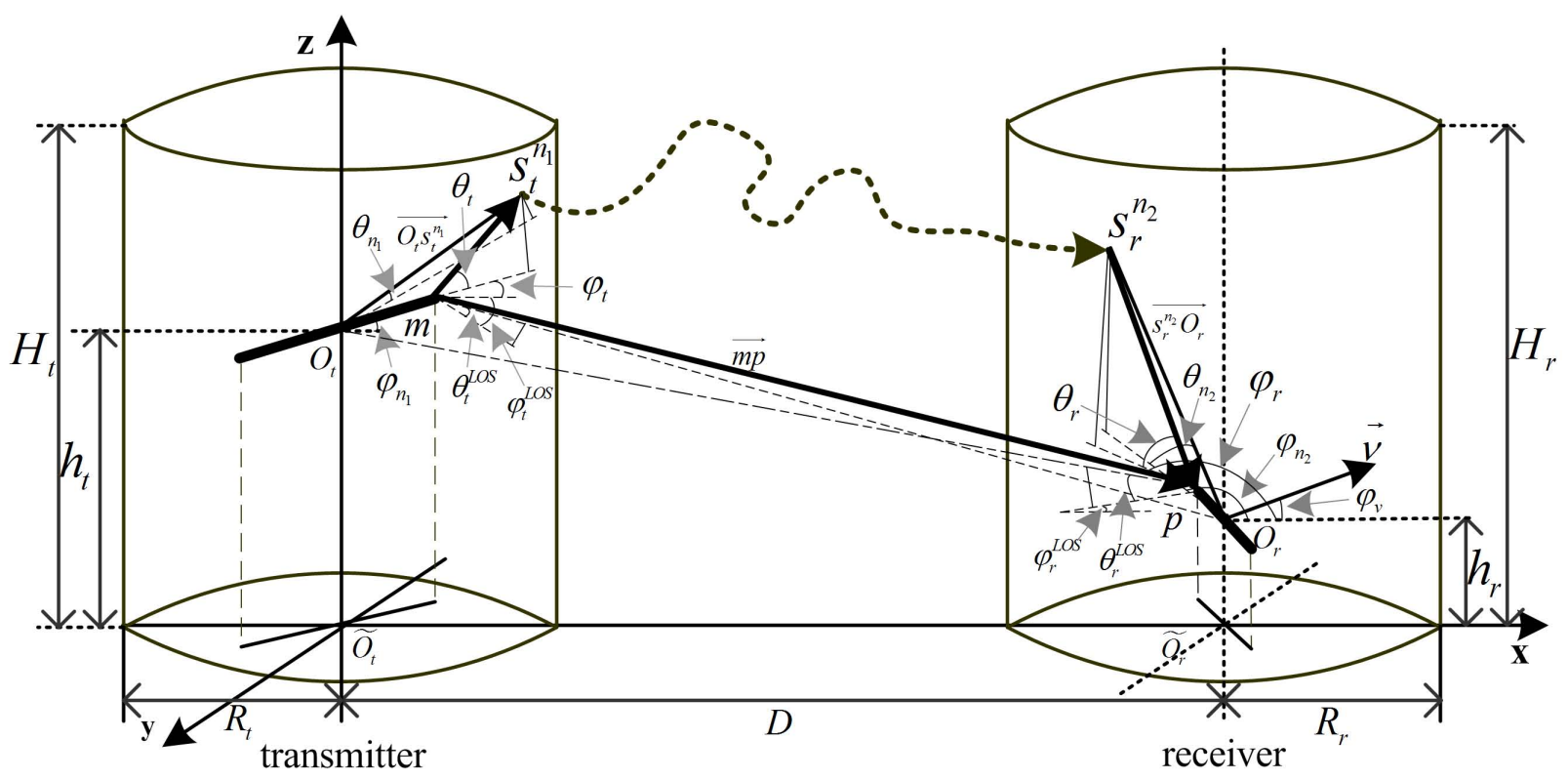

Fig. 2. A 3D GBSM channel model for massive MIMO channels.

( $\lambda$ is wavelength). The channel impulse correlation coefficient is assumed to be 0.9 . Scatters are supposed uniformly distributed on the side surface of the cylinder, whose radius is $R$. The radius of the visible sphere is $r$. To simplify the calculations, the three-dimensional graphics can be expanded into a twodimensional image. An approximate value can be got

$$
r>R \times 2.7856 .
$$

\section{3D GBSM Channel Model For Massive MiMO CHANNELS}

A 3D GBSM channel model for 5G massive MIMO channels is proposed in this section. To the best of our knowledge, it is the first geometrical model for describing the nonstationary property in massive MIMO channel models. As illustrated in Fig. 2, the 3D two-cylinder scattering model contains $M_{t}$ transmitting antenna elements and $M_{r}$ receiver antenna elements. All antennas are omnidirectional and are numbered as $m=1,2 \ldots, M_{t}, p=1,2 \ldots, M_{r}$, respectively. Scatters are randomly distributed in space without considering the length of the path traveled. Each scatter can be determined by two parameters: azimuth angle and elevation angle. Path length is compensated by a time delay. As can be seen from the propagation scenario in Fig. 2, there are two sorts of propagation path: line-of-sight (LOS) and double-bounced propagation. The double-bounced propagations are achieved by the equivalent scatters distributed on the cylindrical surfaces of both the transmitter and the receiver. Different from the physical scatters, an equivalent scatter may include several physical scatters whose delays and angle domains are irresolvable. The first bounces occur when the waves propagate from the transmitting antenna elements to the equivalent scatters at the source side. Similarly, the waves propagate from the equivalent scatters at the destination side to the receiving antenna elements are the last bounces. The propagations from the equivalent scatters at the source side to those at the destination side are abstracted as virtual links. The disappearing and restructuring processes are not considered in the virtual link, because each received path at the destination comes from the source. The scatters on the two cylinders are randomly paired to simulate the uncertain real environment. Equivalent scatters are characterized by amplitude, delay, random phase and so on. With the time goes by, the values of all the parameters update. It is worth noting that in order to significantly reduce the complexity in the 3D theoretical GBSM, other bounced paths are neglected because they show similar channel statistics. Only the geometry of LOS components and the doublebounced two-cylinder model is illustrated in Fig. 2.

In order to simplify the analysis, a uniform linear antenna array with $M_{t}=M_{r}=2$ is adopted as an example. The antennas in proposed model can be extended to arbitrary numbers and the two antenna arrays can be constructed by any shapes. The two cylinders are located on a XoY plane in a 3D coordinating system, and the antenna arrays are parallel to the XoY plane. It is assumed that there are $N_{t}$ equivalent scatters lie on the surface of cylinder at the source side, the radius of the cylinder is denoted by $R_{t}$ and the $n_{1}$ th $\left(n_{1}=1, \ldots, N_{t}\right)$ equivalent scatter is denoted by $s_{t}^{n_{1}}$. Similarly, there are $N_{r}$ equivalent scatters lie on the surface of cylinder at the destination side, where the radius of the cylinder is $R_{r}$ and $n_{2}$ th $\left(n_{2}=1, \ldots, N_{r}\right)$ equivalent scatter is denoted by $s_{r}^{n_{2}}$. Note that the reasonable assumptions $D \gg \max \left(R_{t}, R_{r}\right)$, and $\min \left(R_{t}, R_{r}\right) \gg \max \left(\delta_{t}, \delta_{r}\right)$ are applied in this theoretical model, where $D$ is the distance between the transmitter and the receiver on the XoY plane and $\delta_{t}, \delta_{r}$ are the minimum inter-element spacings of the antenna arrays at the transmitter and the receiver, respectively. The position of each scatter 
on the two cylinders is determined by the azimuth angle and the elevation angle. The azimuth and elevation angles of the scatters in the source side are donated by $\varphi_{n_{1}}$ and $\theta_{n_{1}}$, respectively, and those in the destination side are denoted by $\varphi_{n_{2}}$, and $\theta_{n_{2}} . H_{t}$ and $H_{r}$ are the heights of the cylinders in the source and destination side, $h_{t}$ and $h_{r}$ are the heights of the antenna array in the source and destination side. It's obvious that $H_{t} \gg h_{t}, H_{r} \gg h_{r}, R_{t} \gg \sqrt{M_{t}} \delta_{t}, R_{r} \gg \sqrt{M_{r}} \delta_{r} . f_{\max }$ is the maximum Doppler frequency. It is assumed that the number of the signal propagation paths is $N$ and each path has a power $P_{n}(n=1,2, \ldots, N)$, and $\sum_{n=1}^{N} \sqrt{P_{n}}=1$. The initial phase $\psi_{0}$ is independent and identically distributed (i.i.d.) random variable with uniform distribution over $[0,2 \pi)$, and the LOS Rician factor is $K$. The geometrical parameters are list in Table I.

The 3D massive MIMO channel model is described by an $M_{t} \times M_{r}$ matrix with complex fading envelopes, $H_{t}=$ $\left[h_{m p}(t)\right]_{M_{t} \times M_{r}}$. The received complex fading envelope between the $m$ th and the $p$ th antenna at the frequency of $f_{c}$ is a superposition of the LoS and the double-bounced components, which can be expressed as

$$
h_{m p}(t)=h_{m p}^{\mathrm{LOS}}(t)+\sum_{n=1}^{N} h_{m p}^{n}(t),
$$

where

$$
\begin{aligned}
h_{m p}^{\mathrm{LOS}}(t) & =\sqrt{\frac{K}{K+1}} \exp \left\{j \left(\psi_{0}+\frac{2 \pi}{\lambda}|\overrightarrow{m p}|\right.\right. \\
& \left.\left.+2 \pi f_{\max } t \frac{\langle\overrightarrow{m p} \cdot \vec{\nu}\rangle}{|\overrightarrow{m p}| \cdot|\vec{\nu}|}\right)\right\},
\end{aligned}
$$

where $\rightarrow$ donates vector, $\langle\cdot\rangle$ donates inner product, $|\cdot|$ donates module and

$$
\begin{aligned}
h_{m p}^{n}(t) & =\sqrt{\frac{P_{n}}{K+1}} \exp \left\{j \left[\psi_{0}+2 \pi f_{\max } t \frac{\left\langle\overrightarrow{s_{r}^{n_{2}} p} \cdot \vec{\nu}\right\rangle}{\left|\overrightarrow{s_{r}^{n_{2}} p}\right| \cdot|\vec{\nu}|}\right.\right. \\
& \left.\left.+\frac{2 \pi}{\lambda}\left(\left|\overrightarrow{m s_{t}^{n_{1}}}\right|+\left|\overrightarrow{s_{r}^{n_{2}} p}\right|+\left|\widetilde{s_{t}^{n_{1}} s_{r}^{n_{2}}}\right|\right)\right]\right\} .
\end{aligned}
$$

\section{Channel Modeling And Simulation Procedure}

\section{A. Channel Modeling Process}

The channel modeling process is described as Alg. 1. The procedure in line 7 is used to achieve the non-stationary property. The purpose of line 8 is to simulate signal propagation path. Finally, the channel matrix can be calculated in the final step.

\section{B. Evaluation of Statistical Properties of the Massive MIMO Channel Mode}

Based on our 3D two-cylinder GBSM channel model, it is possible to derive the corresponding statistical properties, including spatial cross-correlation function (CCF), temporal autocorrelation function (ACF), Doppler power frequency density (DSP) and condition number. With these statistical properties, the performance of the proposed channel model can be evaluated.

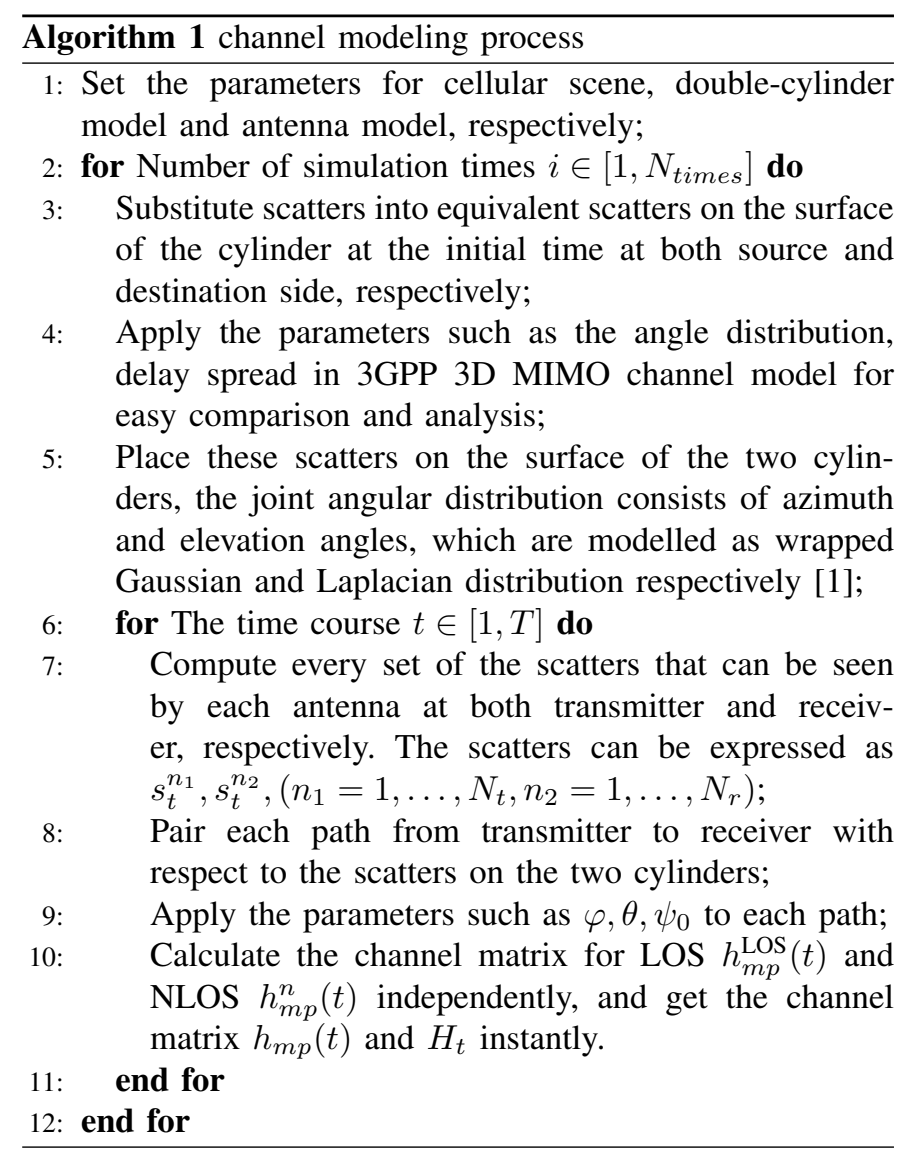

\section{1) Spatial Cross-Correlation Function}

Spatial correlation characteristics and time-related characteristics simultaneously exist in any fading channels. Spatial correlation is used to evaluate correlation between different antennas, and time-related correlation reflects the autocorrelation characteristics of a single receiving antenna at different time. The correlations in fading channels affect each other. In order to analyze the spatial correlation or the time-related correlation easily, when analyzing the spatial correlation, we do not take the time-related correlation into consideration. By setting $\tau=0$, the spatial-temporal correlation function reduces to the spatial cross-correlation $(\mathrm{CCF})$ function

$$
\begin{aligned}
& R_{h_{m p}, h_{m^{\prime} p^{\prime}}}(t)=\frac{E\left[h_{m p}(t) h_{m^{\prime} p^{\prime}}^{*}(t)\right]}{\sqrt{E\left[\left|h_{m p}(t)\right|^{2}\right] E\left[\left|h_{m^{\prime} p^{\prime}}(t)\right|^{2}\right]}} \\
& =R_{h_{m p}^{\mathrm{LOS}}, h_{m^{\prime} p^{\prime}}^{\mathrm{LOS}}}(t)+R_{h_{m p}^{\mathrm{NLOS}}, h_{m^{\prime} p^{\prime}}^{\mathrm{NLOS}}}(t),
\end{aligned}
$$

where $(\cdot)^{*}$ denotes the complex conjugate operation and $E[\cdot]$ represents the expectation value,

$$
\begin{aligned}
& R_{h_{m p}^{\mathrm{LOS}}, h_{m^{\prime} p^{\prime}}^{\mathrm{LOS}}}(t)=\frac{K}{K+1} \exp \left\{j \frac{2 \pi}{\lambda}\left(|\overrightarrow{m p}|-\left|\overrightarrow{m^{\prime} p^{\prime}}\right|\right)\right\} \\
& \times \exp \left\{2 \pi f_{\max } t\left(\frac{\langle\overrightarrow{m p} \cdot \vec{\nu}\rangle}{|\overrightarrow{m p}| \cdot|\vec{\nu}|}-\frac{\left\langle\overrightarrow{m^{\prime} p^{\prime}} \cdot \vec{\nu}\right\rangle}{\left|\overrightarrow{m^{\prime} p^{\prime}}\right| \cdot|\vec{\nu}|}\right)\right\}
\end{aligned}
$$


TABLE I

DEFINITION OF THE PARAMETERS USED IN THE PROPOSED MODEL

\begin{tabular}{|c|c|}
\hline Parameters & Meaning \\
\hline$O_{t}, O_{r}, \widetilde{O}_{t}, \widetilde{O}_{r}$ & The center of the antenna array and their projection on XoY plane of transmitter and receiver, respectively. \\
\hline$H_{t}, H_{r}, R_{t}, R_{r}$ & The heights and radii of cylinders at transmitter and receiver, respectively. \\
\hline$D$ & The distance between transmitter and receiver on XoY plane. \\
\hline$h_{t}, h_{r}, \delta_{t}, \delta_{r}$ & The heights of the antenna array and the inter-element distances at the transmitter and receiver, respectively. \\
\hline $\begin{array}{c}M_{t}, M_{r}, m, p \\
m=1, \ldots, M_{t}, p=1, \ldots, M_{r}\end{array}$ & The numbers of the antennas at transmitter and receiver, respectively. \\
\hline$N_{t}, N_{r}$ & The numbers of the scatters at transmitter and receiver, respectively. \\
\hline $\begin{array}{c}s_{t}^{n_{1}}, s_{t}^{n_{2}} \\
n_{1}=1, \ldots, N_{t}, n_{2}=1, \ldots, N_{r}\end{array}$ & The specific scatter at transmitter and receiver, respectively. \\
\hline$\varphi_{n_{1}}, \theta_{n_{1}}, \varphi_{n_{2}}, \theta_{n_{2}}$ & $\begin{array}{l}\text { The azimuth angle and elevation angle with respect to } O_{t}, O_{r} \text { at transmitter and receiver, respectively. } \\
\overrightarrow{O_{t} s_{t}^{n_{1}}}=\left[R_{t} \cos \varphi_{n_{1}}, R_{t} \sin \varphi_{n_{1}}, R_{t} \cos \theta_{n_{1}}\right], \overrightarrow{s_{t}^{n_{2} O_{r}}}=\left[R_{r} \cos \varphi_{n_{2}}+D, R_{r} \sin \varphi_{n_{2}}, R_{r} \cos \theta_{n_{2}}\right] \text {. }\end{array}$ \\
\hline$\vec{\nu}, \nu, \varphi_{\nu}$ & $\begin{array}{l}\text { The velocity vector for the receiver, the size and direction with respect to } O_{r} \text { are } \nu \text { and } \varphi_{\nu} \text {, } \\
\qquad \vec{\nu}=\left[\nu \cos \varphi_{\nu}, \nu \sin \varphi_{\nu}, 0\right] .\end{array}$ \\
\hline$f_{\max }$ & The maximum Doppler frequency shift. \\
\hline $\overrightarrow{m s_{t}^{n}}, \varphi_{t}, \theta_{t}$ & The direction vector from antenna $m$ to scatter $s_{t}^{n_{1}}$, including azimuth angle and elevation angle. \\
\hline $\overrightarrow{s_{r}^{n_{2}} p}, \varphi_{r}, \theta_{r}$ & The direction vector from scatter $s_{r}^{n_{2}}$ to antenna $p$, including azimuth angle and elevation angle. \\
\hline $\overrightarrow{m p}, \varphi_{t}^{\mathrm{LOS}}, \theta_{t}^{\mathrm{LOS}}, \varphi_{r}^{\mathrm{LOS}}, \theta_{r}^{\mathrm{LOS}}$ & $\begin{array}{l}\text { The LOS direction vector from antenna } m \text { to antenna } p \text {, } \\
\text { including azimuth angles and elevation angles at the transmitter and receiver, respectively. }\end{array}$ \\
\hline $\bar{K}$ & Rice factor for LOS. \\
\hline$\psi_{0}$ & The initial phase of the path. \\
\hline$P_{n}(n=1,2, \ldots, N)$ & The power of each path. \\
\hline$r_{t}, r_{r}$ & stationary property at the transmitter and receiver, respectively. \\
\hline
\end{tabular}

and

$$
\begin{array}{r}
R_{h_{m p}^{\mathrm{NLOS}}, h_{m^{\prime} p^{\prime}}^{\mathrm{NLOS}}}(t)=\sqrt{\frac{P}{K}} \times \sum_{n=1}^{N} \sum_{m=1}^{M} \exp \left\{j \frac{2 \pi}{\lambda} \Delta s\right. \\
\left.+2 \pi f_{\max } t\left(\frac{\left\langle\overrightarrow{s_{r}^{n_{2}} p} \cdot \vec{\nu}\right\rangle}{\left|\overrightarrow{s_{r}^{n_{2}} p}\right| \cdot|\vec{\nu}|}-\frac{\left\langle\overrightarrow{s_{r}^{m_{2}} p^{\prime}} \cdot \vec{\nu}\right\rangle}{\left|\overrightarrow{s_{r}^{m_{2}} p^{\prime}}\right| \cdot|\vec{\nu}|}\right)\right\} .
\end{array}
$$

\section{2) Temporal Autocorrelation Function}

Autocorrelation function expresses the dependencies of each other in the same process between different moments. Here, by setting $m^{\prime}=m, p^{\prime}=p$, we can get temporal autocorrelation function

$$
\begin{aligned}
R_{h_{m p}}(\tau) & =\frac{E\left[h_{m p}(t) h_{m p}^{*}(t-\tau)\right]}{\sqrt{E\left[\left|h_{m p}(t)\right|^{2}\right] E\left[\left|h_{m p}^{*}(t-\tau)\right|^{2}\right]}} \\
& =R_{h_{m p}^{\mathrm{LOS}}(\tau)+R_{h_{m p}^{\text {NLOS }}}(\tau),}
\end{aligned}
$$

where LOS component is

$$
R_{h_{m p}^{\operatorname{LOS}}}(\tau)=\frac{K}{K+1} \exp \left\{2 \pi f_{\max } \tau \frac{\langle\overrightarrow{m p} \cdot \vec{\nu}\rangle}{|\overrightarrow{m p}| \cdot|\vec{\nu}|}\right\},
$$

and NLOS component is

$$
\begin{aligned}
& R_{h_{m p}^{\text {NLOS }}}(\tau)=\sum_{n=1}^{N} \sum_{m=1}^{M} \sqrt{\frac{P}{K}} \exp \left\{-2 \pi f_{\max } \tau \frac{\left\langle\overrightarrow{s_{r}^{m_{2}} p} \cdot \vec{\nu}\right\rangle}{\left|\overrightarrow{s_{r}^{m_{2}} p}\right| \cdot|\vec{\nu}|}\right. \\
& \left.+j \frac{2 \pi}{\lambda} \Delta s+2 \pi f_{\max } t\left(\frac{\left\langle\overrightarrow{s_{r}^{n_{2}} p} \cdot \vec{\nu}\right\rangle}{\left|\overrightarrow{s_{r}^{n_{2}} p}\right| \cdot|\vec{\nu}|}-\frac{\left\langle\overrightarrow{s_{r}^{m_{2}} p} \cdot \vec{\nu}\right\rangle}{\left|\overrightarrow{s_{r}^{m_{2}} p}\right| \cdot|\vec{\nu}|}\right)\right\} .
\end{aligned}
$$

\section{3) Doppler Power Frequency Density}

Channel characteristics change as the signal propagating, resulting in selective fading. A time-selective fading channel is caused by the relative motion between the mobile station and the base station, or caused by the moving of objects in the path. Different antennas on the same array will experience different Doppler shifts because of the spherical propagation of the signal. Therefore, Doppler frequencies may vary on the antenna array. Applying the Fourier transform to the spatialtemporal correlation function, we can obtain the corresponding Doppler PSD

$$
\begin{aligned}
S_{h_{m p}, h_{m^{\prime} p^{\prime}}}\left(f_{D}\right) & =\mathscr{F}\left\{R_{h_{m p}, h_{m^{\prime} p^{\prime}}}(\tau)\right\} \\
& =\int_{-\infty}^{+\infty} R_{h_{m p}, h_{m^{\prime} p^{\prime}}}(\tau) e^{-j 2 \pi f_{D} \tau} d \tau,
\end{aligned}
$$

where $f_{D}$ is the Doppler frequency, $\mathscr{F}$ donates Fourier transform, and

$$
\begin{aligned}
& S_{h_{m p}, h_{m^{\prime} p^{\prime}}}\left(f_{D}\right)=\mathscr{F}\left\{R_{h_{m p}^{\mathrm{LOS}}, h_{m^{\prime} p^{\prime}}^{\mathrm{LOS}}}(\tau)\right\}+\mathscr{F}\left\{R_{h_{m p}^{\mathrm{NLOS}}, h_{m^{\prime} p^{\prime}}^{\mathrm{NLOS}}}(\tau)\right\} \\
& =\mathscr{F}\left\{R_{h_{m p}^{\mathrm{LOS}}, h_{m^{\prime} p^{\prime}}^{\mathrm{LOS}}}(\tau)\right\}+\mathscr{F}\left\{R_{m m^{\prime}}^{T}(\tau)\right\} \odot \mathscr{F}\left\{R_{p p^{\prime}}^{R}(\tau)\right\},
\end{aligned}
$$

where $\odot$ donates the convolution.

\section{4) Condition Number}

Condition number is a key measurement of the correlation of the channel matrix [3]. If it has higher correlation, there will be larger condition number. The condition number is related to the eigenvalue of the channel matrix.

$$
c(d B)=20 \log _{10} \frac{\lambda_{\max }(H)}{\lambda_{\min }(H)},
$$

where $\lambda_{\max }$ represents the maximum eigenvalue and $\lambda_{\min }$ represents the minimum eigenvalue.

\section{RESUlts And Discussions}

This section presents simulated results based on the proposed model in Section III. The results are analyzed and compared with the 3GPP 3D channel model. The carrier 


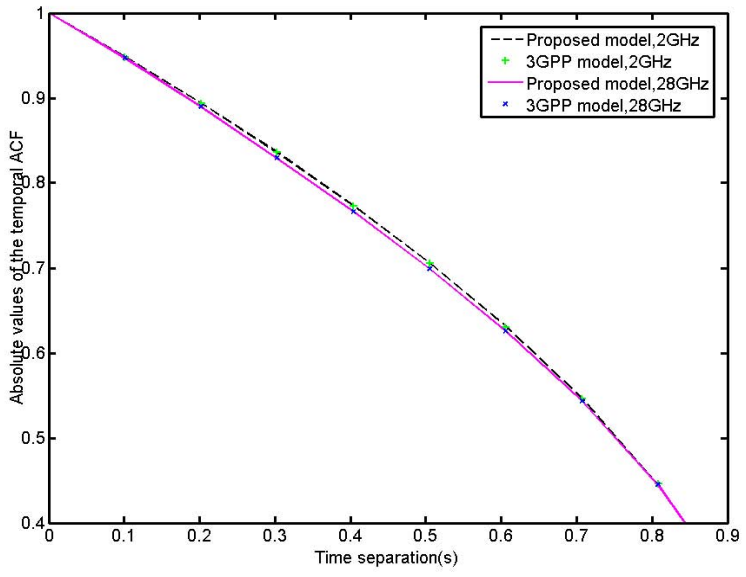

Fig. 3. The absolute value of the ACF.

frequencies of $2 \mathrm{GHz}$ and $28 \mathrm{GHz}$ are included in the simulation separately. The distance between the base station and the mobile station is $D=200 \mathrm{~m}$. The minimum spacing between two antennas is $\delta=\lambda / 2$. The radii of the cylinders on both sides are $R=25 \mathrm{~m}$, and the radii of the spheres used for the non-stationary properties are $r=70 \mathrm{~m}$. The heights of antenna arrays at the base and mobile station are $h_{T}=32 \mathrm{~m}$ and $h_{R}=1.5 \mathrm{~m}$, respectively. The values and distributions of parameters used in this paper come from the 3GPP standard [1].

The absolute values of the temporal ACF are analyzed in Fig. 3. The figure shows that the temporal ACF decreases lower as the time pass. From the figure we observe that the proposed model has similar change trend with the 3GPP model both in $2 \mathrm{GHz}$ and $28 \mathrm{GHz}$. It is because scatters in proposed model and 3GPP model update every moment along the time axis, part of the scatters remained while part of the scatters be replaced by new scatters, the difference between the two models is the number of scatters can be seen, it costs a slight difference.

By setting time to zero, the impacts of the absolute value spatial correlation function are depicted in Fig. 4. Decreasing trends can be observed while the normalized antenna spacing increasing at the receiver side. Spatial correlation with instability caused by the massive antenna elements, the larger the distance between two antennas, the lower the spatial correlation they have. There are not big difference between $2 \mathrm{GHz}$ and $28 \mathrm{GHz}$ because they have the same antenna positions.

As the Doppler PSD is derived from the Fourier transform of corresponding temporal ACF, Fig. 5 shows the Doppler PSDs of the proposed 3D model in both $2 \mathrm{GHz}$ and $28 \mathrm{GHz}$. Because of the non-stationary massive MIMO channel model on the time axis, the PDFs of Doppler frequency at a time instant vary instead of symmetrical with respect to 0 . The physical reason is that the relative movements of the receiving side receives the signal from different directions, and the larger components of the received signal power are from LOS components and the

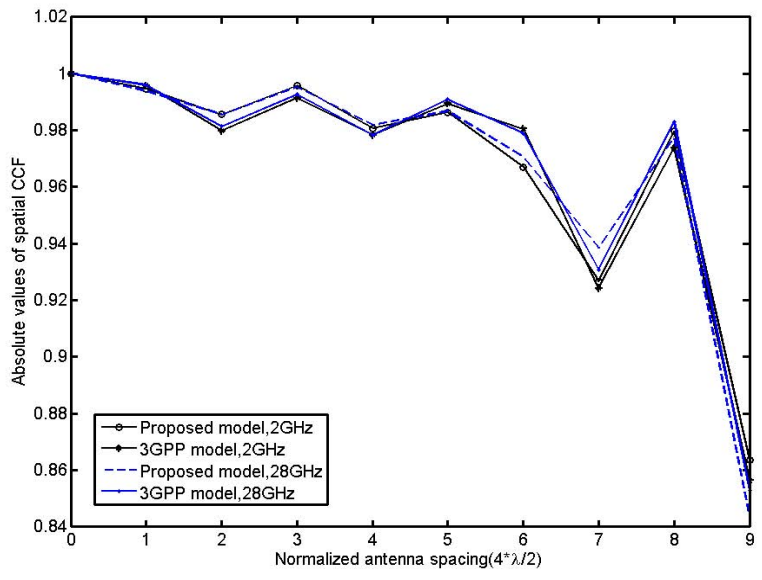

Fig. 4. The absolute value of the CCF.

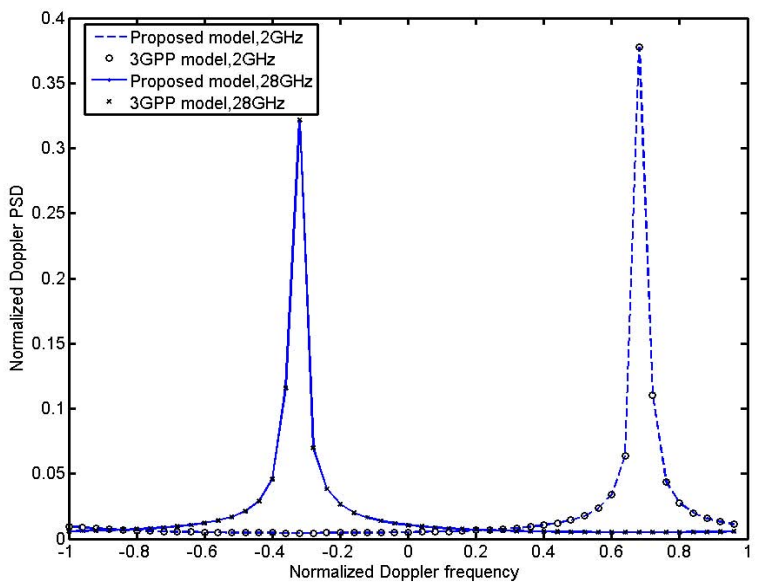

Fig. 5. The normalized Doppler PSD.

static components in the real scattering environment. Doppler PSDs depend on the wavelength and time domain, so they are different in $2 \mathrm{GHz}$ and $28 \mathrm{GHz}$.

A comparison of condition numbers between the $2 \mathrm{GHz}$ and $28 \mathrm{GHz}$ models is shown in Fig. 6. Stronger correlations are observed in 3GPP model than the proposed model due to the fact that clusters have higher probabilities to be correlated in 3GPP model. And stronger correlations are observed in the $28 \mathrm{GHz}$ model than the $2 \mathrm{GHz}$ model, antenna spacing with $2 \mathrm{GHz}$ is larger than $28 \mathrm{GHz}$, so it has higher probabilities to see new scatters than $28 \mathrm{GHz}$. But the difference is small because scatters' locations are randomly distributed in space.

Through the above analysis, the proposed channel model can be seen to be effective. Thanks to the GBSM-based modeling method, there are several advantages over the other modeling methods. Firstly, the proposed model has an intuitive geometrical meaning, which is easy for academics to understand and use. Secondly, the parameters in this model are easy to obtain from the measurements in real environments. 


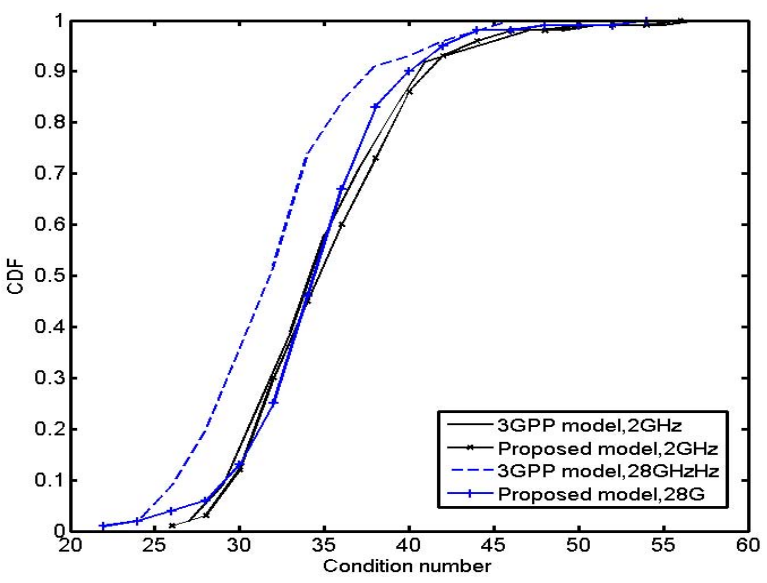

Fig. 6. Conditional number.

For example, the heights of the cylinders can be got from the elevation range of the signal in vertical plane. Thirdly, the proposed model has good applicability, and it is easy to fit kinds of experimental data. Finally, the proposed model has good compatibility with the general channel model standards.

\section{CONCLusions}

For the large antenna array employed in 5G, there will be three important properties in the massive MIMO model: 3D MIMO, spherical wave effect, and especially the nonstationary property. Considering these three properties, a 3D geometry-based stochastic model for 5G massive MIMO channels is proposed in this paper. Using the equivalent scatters which lie on the side surface of the cylinder, it's convenient to simulate the practical scatters. Most importantly, a geometry method is used to achieve non-stationary property. The visible range of each antenna is denoted by a sphere with itself in the center. In order to evaluate the validity of the proposed model, the statistical properties have been derived and investigated. Compared with the 3GPP 3D MIMO channel model, the proposed model have close agreements in the statistical properties with 3GPP channel model. The proposed 3D GBSM massive MIMO channel model is effective to evaluate the 5G communication systems. The proposed model is easy to implement, and provides another effective solution for the modeling of $5 \mathrm{G}$ massive MIMO channels.

\section{ACKNOWLEDGMENT}

This work is supported in part by the National Natural Science Foundations of CHINA (Grant No. 61271279, and 61201157), the National 863 plans project (Grant No. 2014AA01A707, and 2015AA011307), the National Science and Technology Major Project (Grant No. 2015ZX03002006), and the Fundamental Research Funds for the Central Universities (Grant No. 3102015ZY038, 3102015ZY039).

\section{REFERENCES}

[1] "Technical specification group radio access networkstudy on 3D channel model for LTE (Rel 12)," 3GPP TR-36.873, Tech. Rep., Sep. 2014.

[2] S. Alamouti, "A simple transmit diversity technique for wireless communications," IEEE J. Sel. Areas Commun., vol. 16, no. 8, pp. 1451-1458, Oct. 1998.

[3] E. Larsson, O. Edfors, F. Tufvesson, and T. Marzetta, "Massive MIMO for next generation wireless systems," IEEE Commun. Mag., vol. 52, no. 2, pp. 186-195, Feb. 2014

[4] C.-X. Wang, F. Haider, X. Gao, X.-H. You, Y. Yang, D. Yuan, H. Aggoune, H. Haas, S. Fletcher, and E. Hepsaydir, "Cellular architecture and key technologies for 5G wireless communication networks," IEEE Commun. Mag., vol. 52, no. 2, pp. 122-130, Feb. 2014.

[5] K. Zheng, S. Ou, and X. Yin, "Massive MIMO channel models: A survey," International Journal of Antennas and Propagation, 2014.

[6] J. Hoydis, S. ten Brink, and M. Debbah, "Massive MIMO in the UL/DL of cellular networks: How many antennas do we need?" IEEE J. Sel. Areas Commun., vol. 31, no. 2, pp. 160-171, Feb. 2013.

[7] L. Lu, G. Li, A. Swindlehurst, A. Ashikhmin, and R. Zhang, "An overview of massive MIMO: Benefits and challenges," IEEE J. Sel. Top. Sign. Proces., vol. 8, no. 5, pp. 742-758, Oct. 2014.

[8] S. Wu, C.-X. Wang, E.-H. Aggoune, M. Alwakeel, and Y. He, "A nonstationary 3-D wideband twin-cluster model for 5G massive MIMO channels," IEEE J. Sel. Areas Commun., vol. 32, no. 6, pp. 1207-1218, June 2014.

[9] A. Kammoun, H. Khanfir, Z. Altman, M. Debbah, and M. Kamoun, "Survey on 3D channel modeling: From theory to standardization," ArXiv Preprint, 2013.

[10] K. Yu and B. Ottersten, "Models for MIMO propagation channels: a review," Wireless Communications and Mobile Computing, vol. 2, no. 7, pp. 653-666, 2002.

[11] "Spatial channel model for multiple input multipleoutput (MIMO) simulations," G. T. S. ,25.996, V11.0.0, Tech. Rep., 2012.

[12] WINNER II interim channel models, IST-WINNER Std., 2006.

[13] F. Wang, X. Cheng, L. Yang, B. Yu, and B. Jiao, "On the achievable capacity of dual-polarized antenna systems in 3D indoor scenarios," pp. 605-610, Aug. 2013.

[14] J. Parsons and A. Turkmani, "Characterisation of mobile radio signals: model description," IEE Proceedings I: Communications, Speech and Vision, vol. 138, no. 6, pp. 549-556, Dec. 1991.

[15] S. Payami and F. Tufvesson, "Channel measurements and analysis for very large array systems at $2.6 \mathrm{GHz}$," pp. 433-437, Mar. 2012.

[16] X. Gao, F. Tufvesson, O. Edfors, and F. Rusek, "Measured propagation characteristics for very-large MIMO at $2.6 \mathrm{GHz}$," pp. 295-299, Nov. 2012. 